\title{
Exosome secretion, including the DNA damage-induced p53-dependent secretory pathway, is severely compromised in TSAP6/Steap3-null mice
}

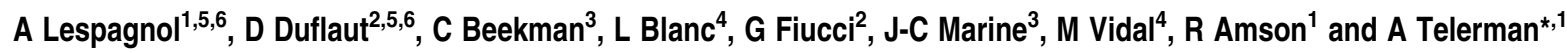

TSAP6 (tumor suppressor-activated pathway 6), also known as Steap3, is a direct p53 transcriptional target gene. It regulates protein secretion, for example translationally controlled tumor protein (TCTP), which is implicated in tumor reversion. In keeping with the latter, we show herein that TSAP6 is a glycosylated protein present in the trans-Golgi network, endosomal-vesicular compartment and cytoplasmic membrane. To further investigate the physiological function of TSAP6, we have generated TSAP6deficient mice. These mice exhibit microcytic anemia with abnormal reticulocyte maturation and deficient transferrin receptor downregulation, a process known to be dependent on exosomal secretion. Moreover, we provide direct evidence that exosome production is severely compromised in TSAP6-null cells. Finally, we show that the DNA damage-induced p53-dependent nonclassical exosomal secretory pathway is abrogated in TSAP6-null cells. Given the fact that exosomes are used as cell-free vaccines against cancer and that they could be involved in the biogenesis and spread of human immunodeficiency virus, it is important to understand their regulation. The results presented here provide the first genetic demonstration that exosome formation is a tightly controlled biological process dependent of TSAP6.

Cell Death and Differentiation (2008) 15, 1723-1733; doi:10.1038/cdd.2008.104; published online 11 July 2008

Tumor suppressor-activated pathway 6 (TSAP6) was initially discovered because differentially regulated following p53 activation $^{1}$ and was later shown to be strongly activated in tumor suppression and reversion, which is why we named it TSAP6, in contrast with other transcripts which are inhibited in their expression (TSIPs). ${ }^{1,2}$ Its promoter contains a functional p53-responsive element. ${ }^{3}$ TSAP6 is part of a family of oxydoreductases with six transmembrane domains ${ }^{4}$ and was recently identified as a ferrireductase (Steap3). ${ }^{5}$ TSAP6 knockdown results in inhibition of apoptosis. ${ }^{3}$ It binds to ${ }^{3}$ and cooperates with $\mathrm{Nix}$, a proapoptotic, BH3-only, Bcl2 familymember and Myt1 kinase, a negative regulator of the $G_{2} M$ transition.

Among the most intriguing functions of TSAP6 is its potential role in the transport of proteins through the nonclassical pathway. TSAP6 is indeed required for the secretion of translationally controlled tumor protein (TCTP), ${ }^{6}$ a key player in tumor reversion. ${ }^{7,8}$

The nonclassical pathway was described following the discovery that proteins such as interleukin $1 \beta$ and galectin could be secreted by cells in the absence of a functional ER-Golgi system. ${ }^{9,10}$ There are different nonclassical export routes and the exosomes are one of them. ${ }^{11}$ The nonclassical export routes are of major importance as they are responsible for the secretion of human immunodeficiency virus (HIV)-tat, Herpes simplex VP22, angiogenic growth factors, inflammatory cytokines and components of the extracellular matrix. ${ }^{11}$ The exosomes have been discovered through the work on the reticulocyte maturation process concomitant with the transferrin receptor (Tf-R) release. ${ }^{12,13}$ The exosomal pathway could be 'hijacked' by certain viruses, and vehiculates HIV. ${ }^{14,15}$ Exosomes are considered as 'vesicles shipping extracellular messages' including proteins such as MHC class I and II, integrins, tetraspanins and heat-shock proteins. Exosomes are important in immunity. ${ }^{16,17}$ Zitvogel et al. ${ }^{18}$ found that exosomes derived from dendritic cells induce an immune response strong enough to eradicate tumors, opening hereby a new treatment strategy: cell-free vaccines. Although it was known that TSAP6 is a p53 target gene and that it regulates the nonclassical pathway of protein secretion, it was only recently highlighted, through a series of elegant experiments by Levine and co-workers, that p53 could control the secretion of exosomes. ${ }^{3,6,19}$ We provide herein the first genetic evidence that secretion of exosomes is TSAP6 dependent and that TSAP6 regulates the p53-dependent nonclassical exosomal protein secretion pathway.

\footnotetext{
${ }^{1}$ Laboratoire de Biotechnologie et Pharmacologie génétique Appliquée (LBPA), UMR 8113, École Normale Supérieure, Cachan, France; ${ }^{2}$ Molecular Engines Laboratories, Paris, France; ${ }^{3}$ Laboratory for Molecular Cancer Biology, VIB-UGent, Technologiepark, Ghent, Belgium and ${ }^{4}$ UMR CNRS 5235, Université Montpellier II, Montpellier, France

*Corresponding author: A Telerman, Laboratoire de Biotechnologie et Pharmacologie génétique Appliquée (LBPA), UMR 8113, École Normale Supérieure, 61 Avenue du Président Wilson, Cachan 94235, France. Tel: +3362089 13 82; Fax: + 331474076 71;

E-mail: adam.telerman@lbpa.ens-cachan.fr and telerman@noos.fr

${ }^{5}$ These authors made equal contributions to this paper.

${ }^{6} \mathrm{PhD}$ students affiliated, respectively, to the Ecole Doctorale Paris VII Diderot and to the Ecole Doctorale de Cancérologie Paris XI.

Keywords: exosome; p53; TCTP; tumor reversion

Abbreviations: TSAP6, tumor suppressor-activated pathway 6; TCTP, translationally controlled tumor protein; MEFs, mouse embryo fibroblasts; PARP, poly (ADPribose) polymerase; TUNEL, terminal deoxynucleotidyl transferase-mediated dUTP nick end-labeling; PHZ, Phenylhydrazine; PFT, Pifithrin

Received 24.10.07; revised 12.5.08; accepted 03.6.08; Edited by KH Vousden; published online 11.7.08
} 


\section{Results}

TSAP6-deficient mice display a microcytic anemia. TSAP6 is located on murine chromosome 1, spans over $38.8 \mathrm{~kb}$ and contains six coding exons. TSAP6 knockout (KO) mice were generated by deleting exons 2 and 3 (Figure 1a). To demonstrate that this mutation results in the complete ablation of Tsap6 expression, we produced a rabbit antiserum directed against a N-terminal peptide of the murine TSAP6. In NIH3T3 cells, the antibodies detect by western blot analysis two major bands of 46 and $52 \mathrm{kDa}$ (Figure 1b) that are strongly downregulated with different TSAP6 siRNAs (data not shown) of which one is exemplified in Figure 1b. Importantly, these two bands are absent in mouse embryo fibroblasts (MEFs) from TSAP6-/- embryos (Figure 1c). The upper band is the result of a glycosylation as it strongly fades away after treatment of the cells with $\mathrm{N}$-glycosidase (Figure 1d). Confocal microscopy shows that the TSAP6 protein is localized at the perinuclear region, colocalizing with the trans-Golgi network (TGN) marker TGN38 (Figure 1e). The punctuated cytoplasmic and plasma membrane staining partially colocalizes with the Tf-R and Early Endosomal protein (EEA1) (Figure 1f; Supplementary Figure 1), suggesting that TSAP6 is expressed in the endosomal compartment. Importantly, this staining is absent in MEFs from the TSAP6-I- embryos (Figure 1e and f). These results suggest that the antibodies are highly specific for the TSAP6 protein and that deletion of exons 2 and 3 leads to a Tsap6-null mutation. The $46-52 \mathrm{kDa}$ TSAP6 protein is expressed at variable levels in tissues of wild-type (WT) mice (Figure 1g). As our previous data suggested that TSAP6 decreases the cellular level of TCTP, we examined the expression of TCTP and Tf-R in Tsap6-null cells. As shown in Figure $1 \mathrm{~h}$ the splenocytes from TSAP6 KO mice accumulate TCTP and the Tf-R, which is consistent with the idea that TSAP6 could regulate protein secretion.

TSAP6-/- mice show a significant splenomegaly (Figure 1i), extramedullary hematopoiesis with an alteration of the architecture, the white pulp being more spread and the red pulp showing small cells irregular in shape and variable in size (Figure 1j). The peripheral blood counts show a pronounced increase in platelets and an increased number of red blood cells (RBC) with a drop in hemoglobin, mean corpuscular volume (MCV) and mean corpuscular hemoglobin ( $\mathrm{MCH}$; Figure 2a). TSAP6-/- reticulocytes and erythrocytes are much smaller, display an abnormal shape and a pronounced osmotic fragility (Figure $2 \mathrm{~b}-\mathrm{e}$ ). Other biochemical parameters tested are within the normal range, besides for a mild hyperglycemia in female TSAP6 KO mice (Supplementary Figure 2).

TSAP6-I- mice exhibit delayed reticulocyte maturation with retention of the Tf-R as a consequence of reduced secretion of exosomes. We next investigated whether the reticulocytes from TSAP6-/- mice go through normal maturation. We first assessed the status of the Tf- $R$ at the surface of reticulocytes from animals that were not treated with phenylhydrazine (PHZ) (Figure 3a). The maturation state of red cells from 13 WT controls and 11 TSAP6 KO mice was analyzed. As shown in Figure 3a the percentage of Tf-R-labeled red cells was higher in TSAP6 KO mice $(7.28 \pm 2.49 \%)$ than in WT mice $(2.60 \pm 0.32 \%)$, reflecting an anemic phenotype (see also Supplementary Figure 3). A May-Grünwald-Giemsa staining of the bone marrow shows an increased number of RBCs precursors (WT: $20 \pm 2 \%$; TSAP6 KO: $37 \pm 8 \%$ ), the erythroblasts, characterized by a round nucleus and a basophilic cytoplasm (Supplementary Figure $3 \mathrm{~A}$ and $\mathrm{B}$ ). The Tf-R was measured in Ter119-positive cells representing the erythroid population. Two populations of Ter119 positive and Tf-R positive cells were identified (Supplementary Figure $3 \mathrm{C}$ and D). R1 represents the population with high levels and R2 the one with low levels of Tf-R. In the bone marrow erythroblasts, the R1 population is significantly higher in KO mice $(46 \%)$ than in WT mice $(30 \%)$. For the peripheral blood reticulocytes, there is also an increase of the R1 population in the TSAP6 KO mice (11\%) versus the WT mice (4.5\%). The R2 population did not differ significantly in the bone marrow erythroblasts and blood reticulocytes between WT and KO mice. Altogether, this might reflect a lag phase in Tf-R expulsion through exosomes during bone marrow erythroblasts and reticulocyte maturation in TSAP6 KO mice, leading to a larger pool of cell surface-associated Tf-R.

Mice were next rendered anemic by PHZ. Reticulocytes were allowed to mature for 3 days, during which they lose an important fraction of the Tf-R. This can be monitored either by FACS (Figure $3 b$ ) or by western blot analysis (Figure 3c). Under normal conditions the Tf-R content becomes progressively weaker. Using such a classical maturation assay, we observed that in WT animals at $t 061.4 \%$ of the reticulocytes are positive for the Tf-R and that this drops to $33.7 \%$ after $48 \mathrm{~h}$ of maturation. In contrast, the TSAP6 KO mice show $78.2 \%$ of Tf-R present on their reticulocytes at $t 0$ and $84.4 \%$ after $48 \mathrm{~h}$ of in vitro maturation (Figure $3 \mathrm{~b}$ ). This was further confirmed by western blot analysis. The TSAP6 protein dimerizes and oligomerizes into higher migrating forms (Figure $3 \mathrm{c}$ and Supplementary Figure 4). On day 3, the $46-52 \mathrm{kDa}$ TSAP6 is almost undetectable in the WT reticulocytes and all the TSAP6 protein is oligomerized. Concomitantly, there is a decreased Tf-R content. In contrast, in the reticulocytes from TSAP6-/- mice the content in Tf-R remains elevated, suggesting retention of $\mathrm{Tf}-\mathrm{R}$ during reticulocyte maturation. In thymocytes, that do not lose Tf-R during a maturation process, no significant differences in surface Tf-R expression could be detected between WT and TSAP $6 \mathrm{KO}$ cells at $4^{\circ} \mathrm{C}$ (Supplementary Table 1). When incubated at $37^{\circ} \mathrm{C}$ for $1 \mathrm{~h}$, $15-25 \%$ of the receptor was internalized leaving a smaller fraction detectable at the cell surface (Supplementary Table 2). Here again, no significant difference was measured between WT and TSAP6 KO thymocytes suggesting that TSAP6 is not involved in Tf-R internalization process. These results reinforce the notion that the relative accumulation of Tf-R at the cell surface of TSAP6 KO reticulocytes is likely due to a defect in exosome secretion.

We next examined the secretion process during the maturation of the reticulocytes but were unable to obtain highly reproducible results due to the lysis of the TSAP6-/RBCs, likely due to their fragility (Figure 2e).

As reported, bone marrow-derived dendritic cells (BMDCs) $)^{17,20}$ also contain Tf-R, that is abundantly secreted by the exosomes. ${ }^{18}$ Interestingly, the Tf-R (type I, CD71) has been described to be the cell entry receptor for mouse 
a

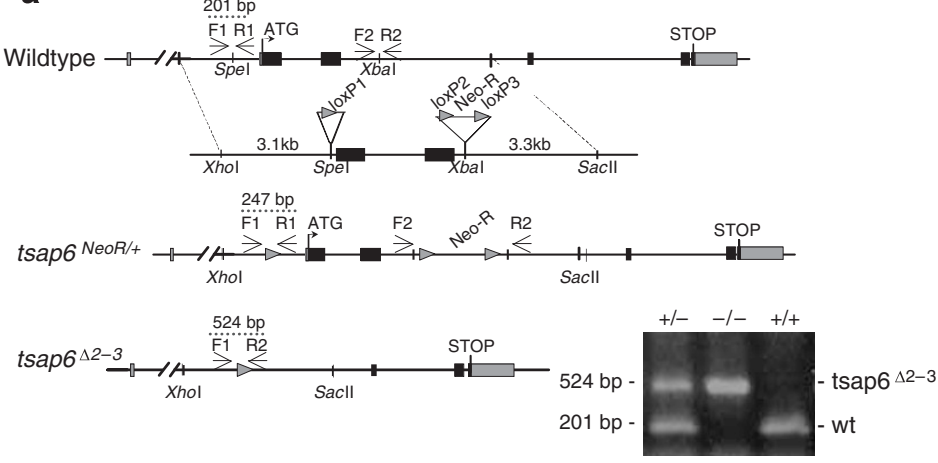

b NIH3T3 Total lysate

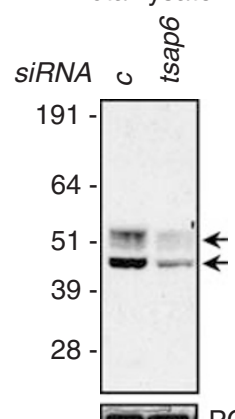

c TSAP6 MEF Total lysate $5 \%$

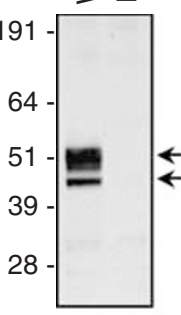

d

NIH3T3

Total lysate e

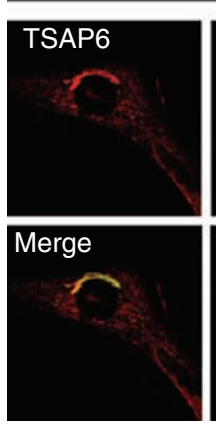

g
TSAP6 KO

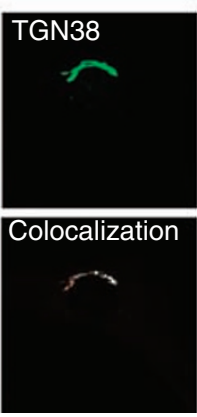

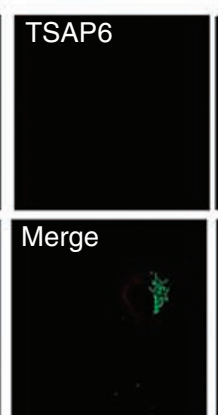

f

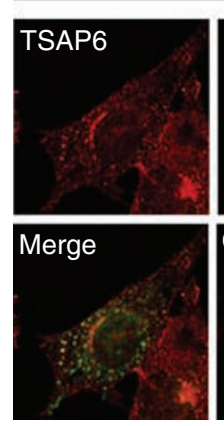

h

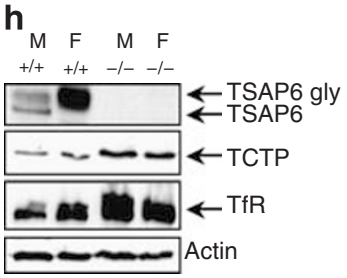

$\mathrm{N}$-glyc - +

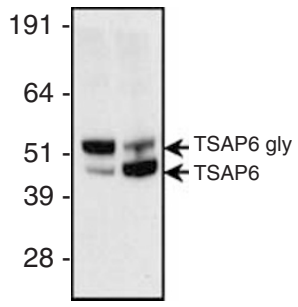

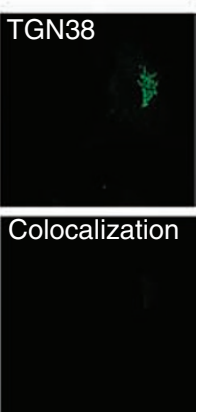

estis

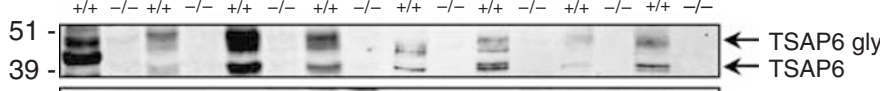

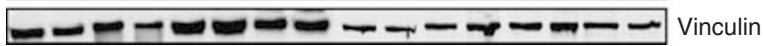

WT

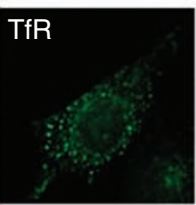

Actin
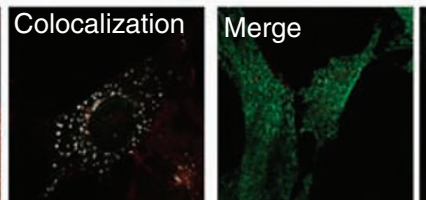

i

\begin{tabular}{|c|c|}
\hline \multicolumn{2}{|c|}{ Ratio of spleen/Body weight } \\
\hline $\begin{array}{c}\text { WT } \\
(n=6)\end{array}$ & $\begin{array}{c}\text { TSAP6 KO } \\
(n=6)\end{array}$ \\
\hline $0.004 \pm 0.0005$ & $0.008 \pm 0.003$ \\
\hline & \\
\hline $10 \quad n \quad 12 \quad \mathrm{~cm}$ & $20 \quad 21 \quad 22 \mathrm{~cm}$ \\
\hline
\end{tabular}

Spleen WT

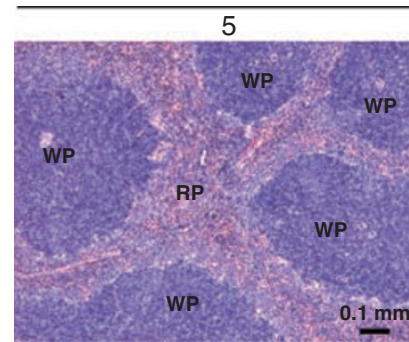

j

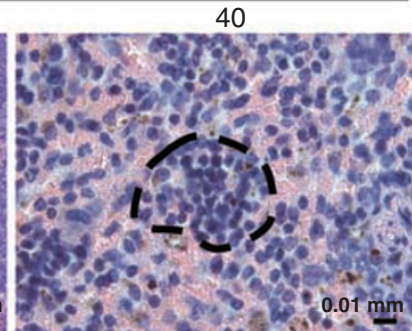

Spleen TSAP6 KO

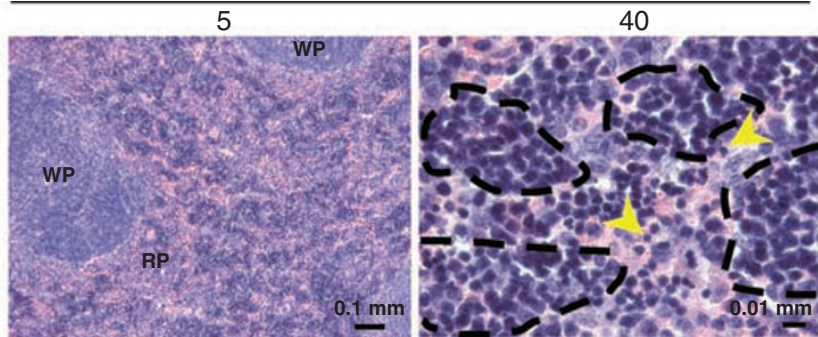

Figure 1 Tumor suppressor-activated pathway 6 (TSAP6) protein and knockout mice. (a) Representation of the murine TSAP6 wild-type allele with the coding exons (black boxes) and the CRE-Lox-targeting construct used to generate the TSAP6 KO mice. Two oligonucleotides were cloned into a PCR-amplified region of the Tsap6 gene: One oligonucleotide containing a single LoxP site and another containing the neomycin resistance gene flanked by LoxP sites. This construct was injected into ES cells and the recombinant clones were used to obtain mice lacking exons 2 and 3. F1, R1 and R2 are the primers used for genotyping. Insert: Three-primer PCR-genotyping strategy performed on genomic DNA from wild-type $(+/+)$, heterozygous $(+/-)$ and TSAP6-null $(-/-)$ mice. (b-d) Western blot analysis using anti-TSAP6 antibodies on protein lysates from (b) NIH3T3 cells transfected with control (c) or TSAP6 siRNA (tsap6); anti-PCNA (proliferating cell nuclear antigen) is used as control for equal loading. (c) wildtype (WT) and TSAP6 knockout (KO) mouse embryo fibroblasts (MEFs); anti-actin is used as control for equal loading. (d) NIH3T3 cells before and after treatment with $\mathrm{N}$-glycosidase (N-glyc). Arrows: 46 and $52 \mathrm{kDa}$ bands of the TSAP6 protein. (e, f) Immunofluorescence analysis of endogenous TSAP6 protein with anti-TSAP6 (red) and colocalization with TGN38 and Tf-R (green), 'merge' in yellow and 'colocalization' in white show a partial codistribution of TSAP6 with the trans-Golgi network and Tf-R in WT MEFs; each confocal microscopy image represents a similar plane through the cell. (g) Western blot analysis of TSAP6 protein expression in different organs $(30 \mu \mathrm{g}$ protein loaded). Antivinculin is used as control for equal loading. (h) Western blot analysis using anti-TSAP6, anti-TCTP and anti-TF-R antibodies on splenocytes from WT ( + I + ) and TSAP6 KO (-I-) male (M) or female (F) mice. Anti-actin is used as control for equal loading. (i) Ratio of spleen to body weight in six WT and six TSAP6 KO female mice. (j) Hematoxylin-eosin staining of spleen sections of WT and TSAP6 KO mice; extramedullar hematopoiesis sites are surrounded by dotted lines; yellow arrows: small erythrocyte precursors of variable size (white pulp, WP; red pulp: RP) 

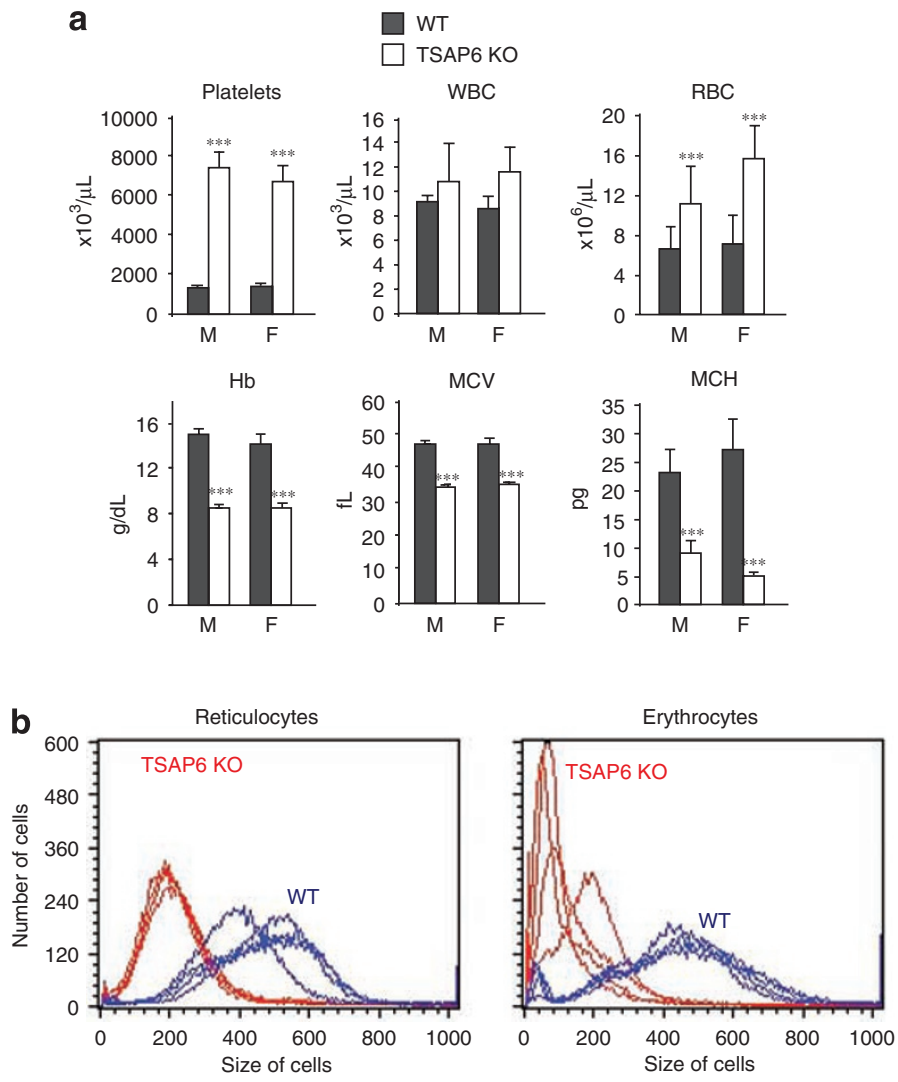
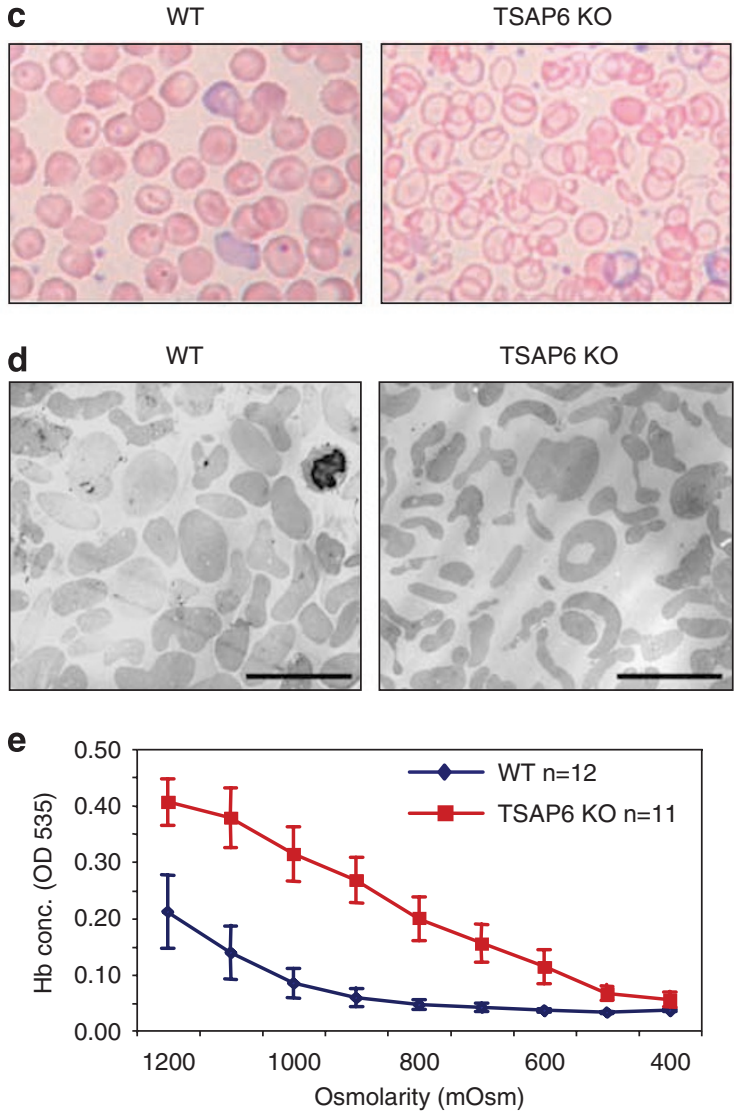

Figure 2 Blood parameters in wild-type (WT) and tumor suppressor-activated pathway 6 (TSAP6) KO mice. (a) Platelets, white blood cells (WBCs), red blood cells (RBCs), hemoglobin ( $\mathrm{Hb})$, mean corpuscular volume (MCV) and mean corpuscular hemoglobin ( $\mathrm{MCH}$ ). The results represent the medium values of six males and six females WT (black bars) versus four males and six females TSAP6 KO (white bars). ${ }^{*} P<0.05$. (b) FACS analysis of reticulocyte (left panel) and erythrocyte (right panel) size in four WT (blue line) and four TSAP6 KO (red line) mice. (c) May-Grünwald-Giemsa staining of peripheral blood smears from WT and TSAP6 KO mice (d) Electron microscopy analysis of WT and TSAP6 KO erythrocytes; bar: $10 \mu \mathrm{m}$. (e) Osmotic fragility analysis of peripheral RBC from TSAP6 KO and WT mice; $n$ : number of mice analyzed

mammary tumor virus (MMTV) ${ }^{21}$ and to play a determining role in the activation of dendritic cells by this retrovirus. ${ }^{22}$ This receptor regulates iron uptake not only in reticulocytes but in most cells. ${ }^{23}$

As shown in Figure 3d, the TSAP6-/- BMDCs express higher levels of Tf-R than their WT counterparts. Most striking is the observation that TSAP6-/- BMDCs secrete $50 \%$ less exosomes than the WT (Figure $3 e$ ). Secretion of the Tf-R by the exosomes of TSAP6-/- mice BMDCs is also severely decreased (Figure $3 f)$. Exosomes migrate at a very specific density and this was confirmed by sucrose gradient centrifugation (Figure $3 \mathrm{~g}$ ). Altogether these results suggest that the TSAP6-/- mice have a strongly impaired secretion of exosomes.

TSAP6 controls exosome secretion in response to DNA damage and p53 activation. As TSAP6 is a p53 target gene, we examined whether or not it functions as a critical mediator of p53-induced biological activities. To this end, mice were irradiated (Figure 4) and p53-induced apoptosis was examined in the spleen by TUNEL and PARP cleavage assays. As expected, a massive induction of apoptosis was detected in the spleen of WT mice (Figure 4a and b). Strikingly, the apoptotic response was significantly attenuated in the spleen from TSAP6-/- animals (Figure $4 a$ and $b$ ). To demonstrate that this phenotype is a consequence of a defect downstream of p53, the expression of p21, a well-known p53 target gene, was examined in irradiated splenocytes. A significant and reproducible increase in p21 expression was observed in WT and TSAP6-deficient cells (Figure 4b and Supplementary Figure $5)$. The difference in the strength of p21 induction could be due to a compensation mechanism activated in some of the animals when the p53 proapoptotic function is compromised - as it is the case here in the absence of TSAP6 - in order to favor p21 induction and cell-cycle exit.

In order to exclude the possibility that Tsap6-deficient splenocytes are more resistant to radiation-induced apoptosis because of a depletion of the radiosensitive cell population(s), splenocytes were further analyzed by FACS for Annexin Vpositive cells and a series of cell-surface markers. In agreement with the above data, the total number of Annexin $\mathrm{V}$-positive cells after irradiation was significantly higher in the WT mice (37\%) than in the TSAP6 KO mice (15.5\%; Figure 4c). Moreover, all subpopulations analyzed, including $B$ cells, T cells or erythrocytes, were less prone to apoptosis in the TSAP6 KO mice than in the WT animals (Figure 4d). More specifically, the Ter119 and CD4-positive cells in the TSAP6 
b

Phenylhydrazine Treatment

a No Phenylhydrazine Treatment

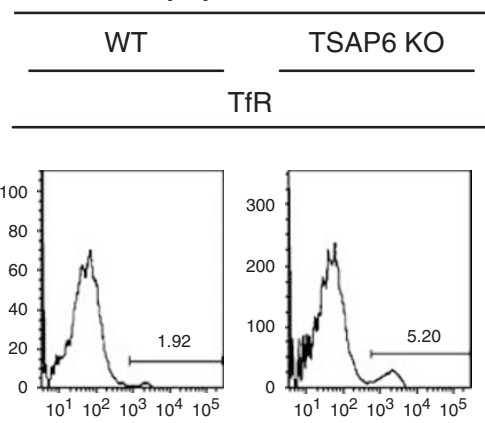

C

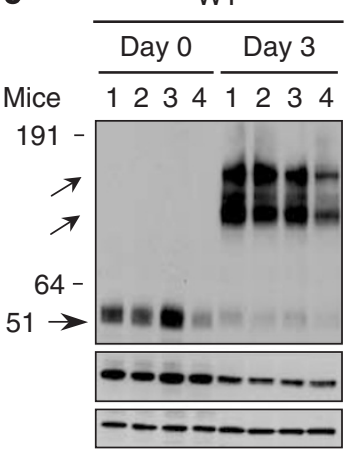

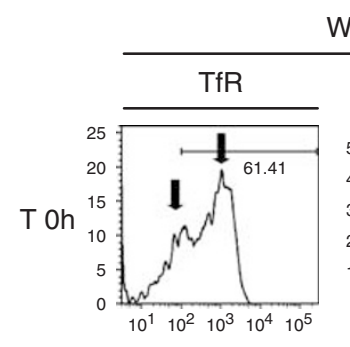

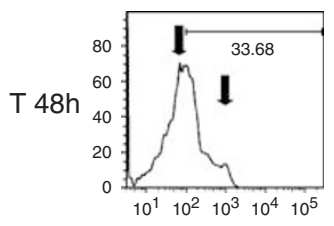

WT
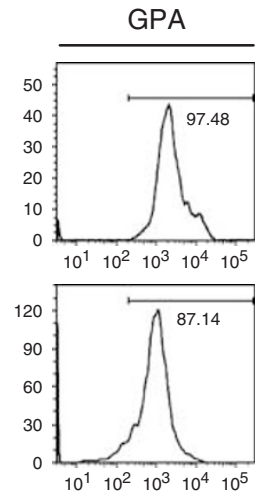

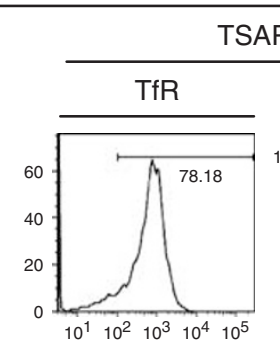

TSAP6 KO

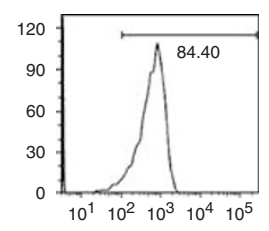

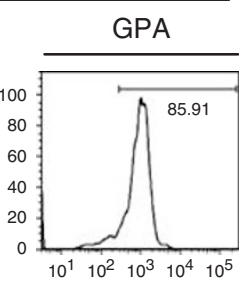

TSAP6 KO
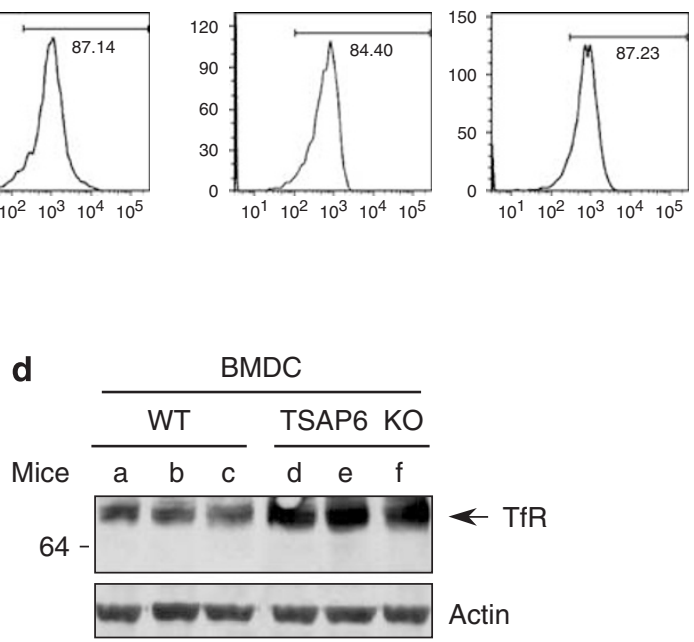

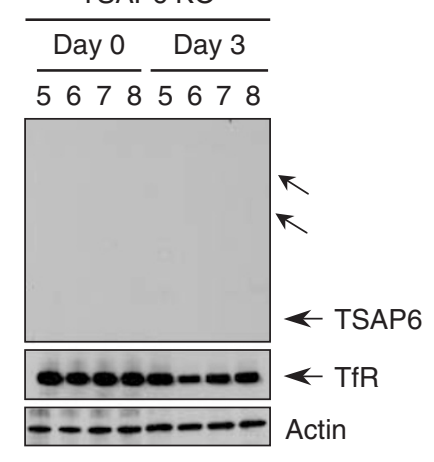

f BMDC exo

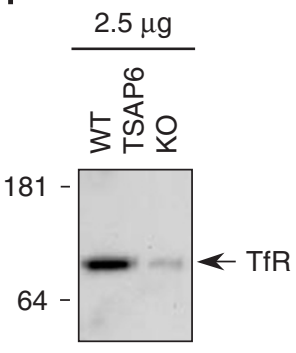

g

Density

$(\mathrm{g} / \mathrm{ml})$

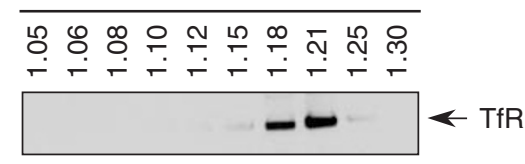

BMDC TSAP6 KO

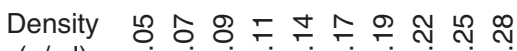

(g/ml)

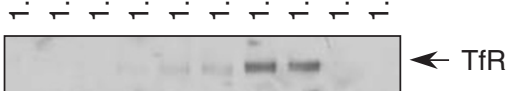

Figure 3 Reticulocyte maturation assay with transferrin receptor (Tf-R) expression, exosome production and Tf-R secretion in wild-type (WT) and tumor suppressoractivated pathway 6 (TSAP6) knockout (KO) mice. (a) FACS analysis with anti-Tf-R antibody of a blood sample from phenylhydrazine-untreated WT (left panel) and TSAP6 KO (right panel) mice. (b) After phenylhydrazine treatment of WT (left panels) or TSAP6 KO (right panels) mice, the presence of Tf-R and Glycophorin A (GPA) was monitored by FACS analysis on freshly isolated reticulocytes (upper panel, $t 0 \mathrm{~h}$ ) and after $48 \mathrm{~h}$ of maturation (lower panel, T48 h). Arrows indicate changes in Tf-R expression during reticulocyte maturation. (c) Western blot analysis with anti-TSAP6 and anti-Tf-R antibodies on the total lysate of purified reticulocytes from WT and TSAP6 KO mice. Differentiation into erythrocytes is analyzed during 3 days. Oblique arrows show potential oligomers of TSAP6, straight arrow shows TSAP6 monomer; anti-actin is used as control for equal loading. (d) Western blot analysis with anti-Tf-R antibodies on the total lysate of bone marrow-derived dendritic cells (BMDCs) from WT and TSAP6 KO mice. (e) Quantitative analysis of exosomal proteins production from $10^{6} \mathrm{BMDC}$ during $6 \mathrm{~h}$. P-values for a significance level at $1 \%$. (f) Western blot analysis of the Tf-R content in $2.5 \mu \mathrm{g}$ of exosomal proteins from BMDCs. (g) Western blot analysis with anti-Tf-R antibodies on fractions collected from $30 \mu \mathrm{g}$ of purified exosomes on a continuous sucrose gradient from BMDC in WT and TSAP6 KO mice

KO mice were extremely resistant to apoptosis. These data indicate that TSAP6 is an essential mediator of p53-induced apoptosis in response to $\gamma$-irradiation.

We next asked whether activation of p53 by DNA damage regulates exosomal protein secretion in a TSAP6-dependent manner. We analyzed the secretion of exosomes in MEFs and BMDCs from WT, and TSAP6-/- mice. The cells were either $\gamma$-irradiated (for the MEFs) or treated with actinomycinD (for the BMDCs). The ratio of secreted exosomal proteins in activated ( $\gamma$-irradiated MEFs or actinomycinD-treated BMDCs) versus resting cells is presented in Figure $5 \mathrm{a}$ and e. When irradiated, WT MEFs secrete twice the amount of exosomal proteins $(1.94 \pm 0.37)$ whereas the TSAP6-/MEFs do not respond significantly $(1.12 \pm 0.29)$ in their exosomal protein secretion (Figure $5 \mathrm{a}$ ). The same quantitative increase in exosomal protein secretion is found in WT BMDCs after they were treated with actinomycinD $(2.65 \pm 0.43)$, although this response is not significant in TSAP6-/- BMDCs 

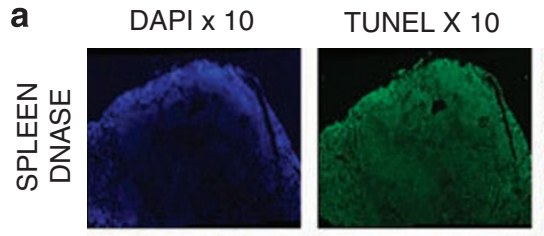

TUNEL X 40
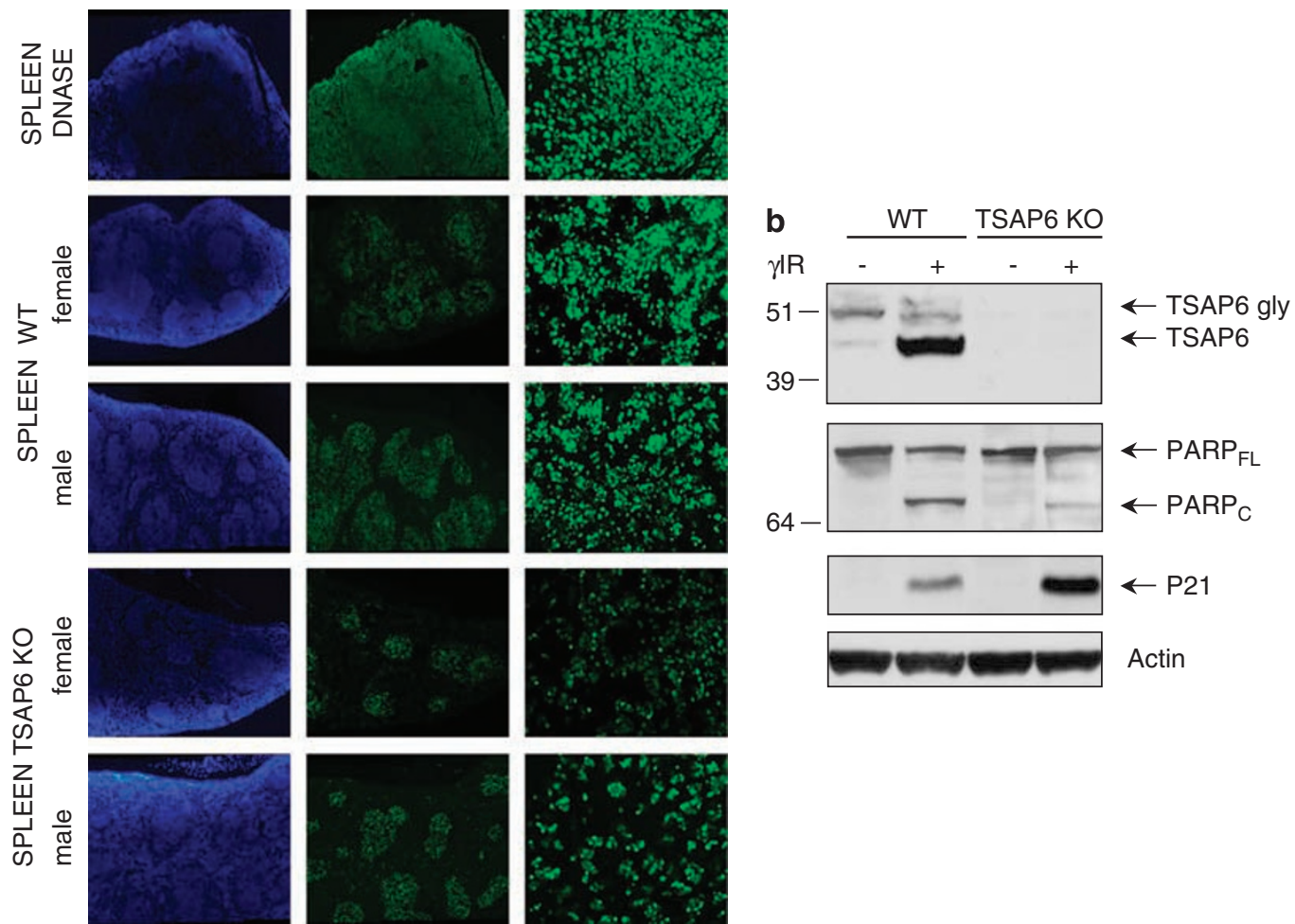

39
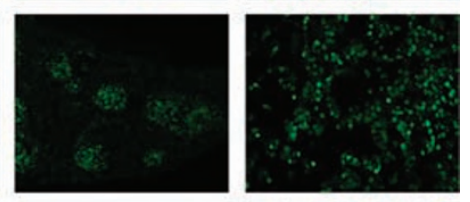

64
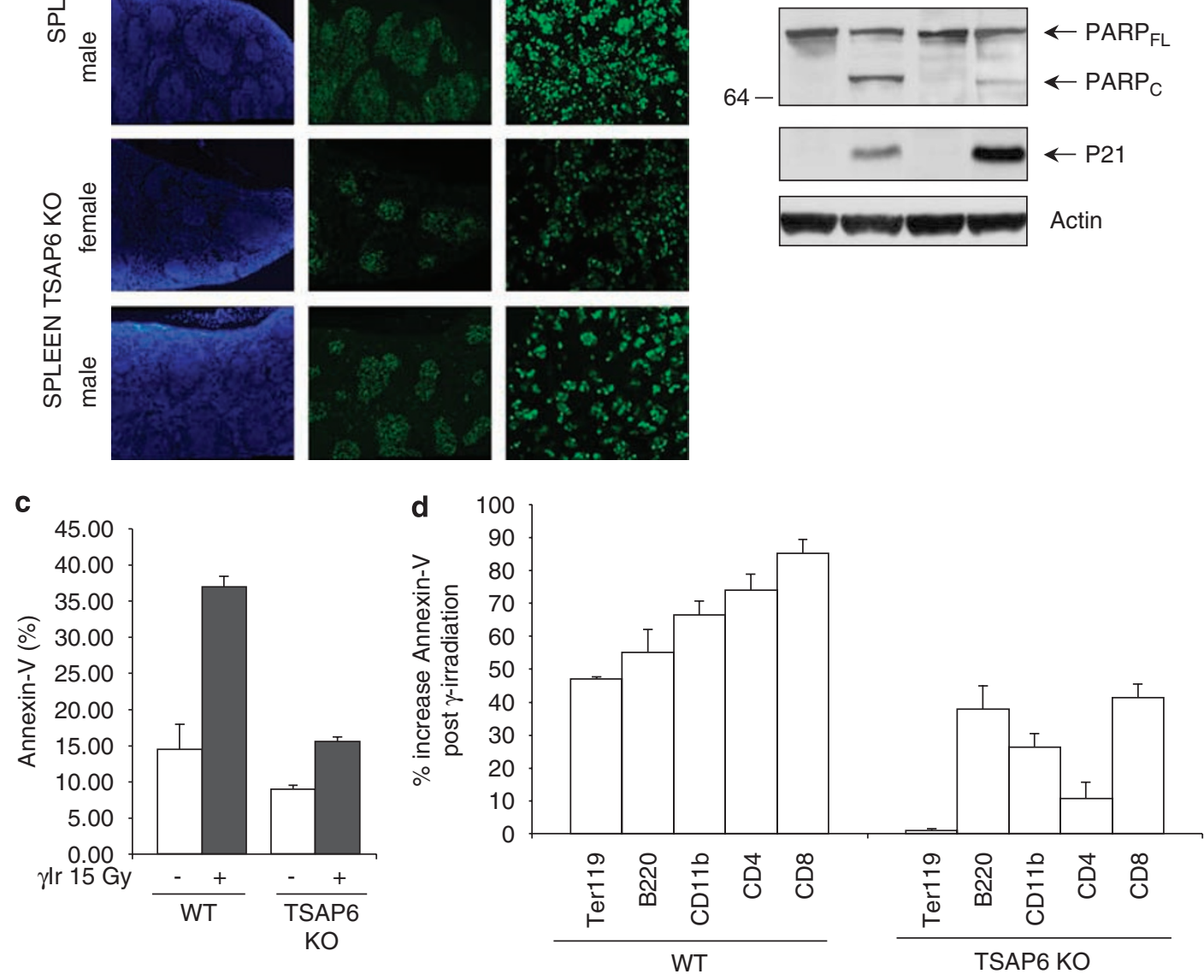

Figure 4 Apoptosis in the spleen of irradiated tumor suppressor-activated pathway 6 (TSAP6) wild-type (WT) and knockout (KO) mice. (a) TUNEL assay on the spleen of TSAP6 WT and KO mice after irradiation with 15 Gy. TUNEL-positive cells are stained in green and all nuclei are stained in blue with DAPI. (b) TSAP6 and P21 protein expression, and PARP cleavage in protein extracts from spleens of WT and TSAP6 KO-irradiated mice; Arrows: PARP full length (FL) and PARP cleaved (C). Anti-actin is used as control for equal loading. (c, d) Apoptosis assay in the spleen of irradiated WT and TSAP6 KO mice: $10 \mathrm{~h}$ after irradiation, the spleen isolated from WT or TSAP6 KO mice were ground and cells were immune-phenotyped by FACS analysis as described in 'Material and Methods'. Apoptosis was determined in each population by Annexin Vpositive labeling. (c) Shows the total percent of Annexin V-positive cells in the spleen before and after irradiation in WT and TSAP6 KO mice. (d) Shows the percent of increase of Annexin V-positive cells for the different lineages tested: red cells (Ter119), B-lymphocytes (B220), granulocytes/monocytes (CD11b), T-lymphocytes (CD4 and CD8), number of animals analyzed: 8

$(1.30 \pm 0.63 ;$ Figure 5e). The difference in the secretion of exosomal proteins between WT and TSAP6-/- cells is highly significant (MEFs WT versus KO $P$-values 0.0001 and BMDCs WT versus KO $P$-values 0.00001 ; Figure $5 \mathrm{a}$ and e). Previous reports quantifying the secretion of exosomes suggest an increase that varies by a factor of $1.2-2 .^{24-26}$ Thus, the present report that TSAP6-/- BMDCs secrete $50 \%$ less exosomes than WT cells, and that activation of p53 by DNA damage increases the secretion by a factor of 2 , is within the limits expected in response to a robust stimulus, that can even be much stronger for one specific protein. $^{26,27}$

When instead of looking at the total amount of secreted exosomal proteins, specific proteins, such as Flotillin, Tsg101 or Galectin-3 are monitored, their secretion increases in MEFs and BMDCs for the WT cells, whereas there is no significant increase detectable in the TSAP6-/- cells (Figure $5 \mathrm{~b}$ and $\mathrm{f}$ ). Of note, the exosomes of TSAP6 KO mice can be inefficient in 
a

Exosomal prot. from irradiated cells Exo prot. from not irradiated cells

$$
\text { WT } 1.94 \pm 0.37 \quad(n=8)
$$

TSAP6 KO $1.12 \pm 0.29 \quad(\mathrm{n}=8)$

$W T$ vs TSAP6 KO, $P$ values $=0.0001$

51

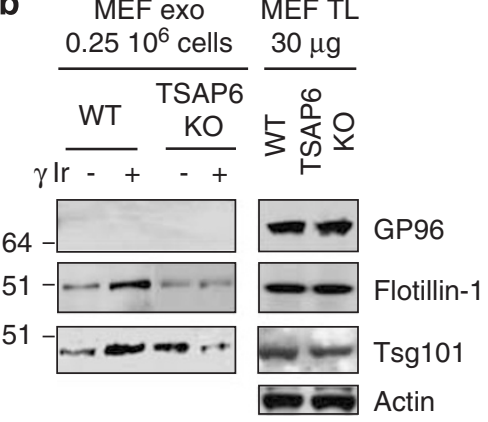

e

$\frac{\text { Exosomal prot. from activated cells }}{\text { Exo prot. from not activated cells }}$

WT $2.65 \pm 0.43$

$(n=6)$

TSAP6 KO $1.30 \pm 0.63 \quad(\mathrm{n}=6)$

$W T$ vs TSAP6 KO, $P$ values $=0.00001$

\section{f}

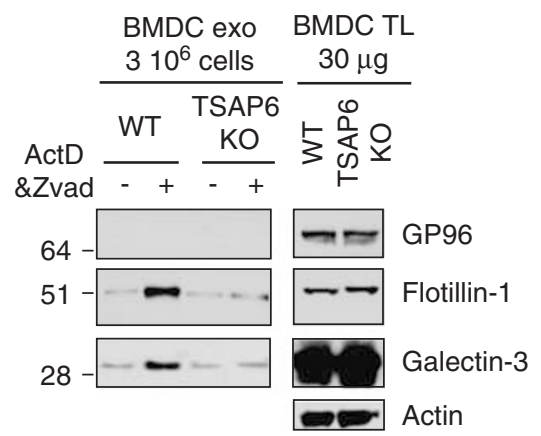

C

Density

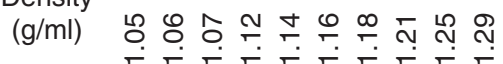

$-\gamma \mathrm{lr}$

$+\gamma \mathrm{lr}$

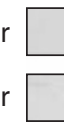

比

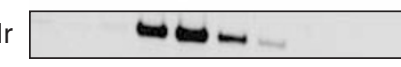

Flotillin-1

Flotillin-1

Density

$(\mathrm{g} / \mathrm{ml})$ 要

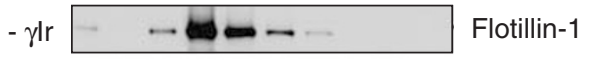

$+\gamma \mathrm{r} \longrightarrow$ Flotillin-1

g

Density BMDC WT

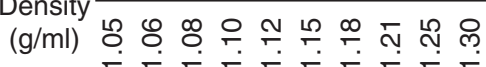

-ActD

-Zvad

+ ActD

+ Zvad

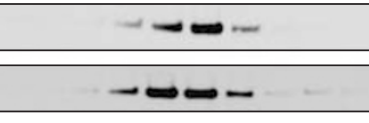

Flotillin-1

Flotillin-1

Density

BMDC TSAP6 KO

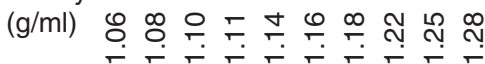

-ActD

- Zvad
+ ActD

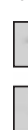

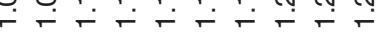

+ Zvad

Figure 5 Exosomal protein secretion in mouse embryo fibroblasts (MEFs) and bone marrow-derived dendritic cells (BMDCs) from wild-type and tumor suppressor-activated pathway 6 (TSAP6) knockout (KO) mice after DNA damage. (a, e) Quantitative analysis of exosomal protein production by $10^{6} \mathrm{MEFs}$ (a) or BMDCs (e). The results are presented as the ratio between exosomal protein production after irradiation (MEFs) or actinomycinD treatmen (BMDCs) versus the exosomal protein production of untreated cells, \pm standard deviation; the difference in exosomal protein production between WT and TSAP6 KO is calculated for $P$-values at a significance level of $1 \%$. ( $b$, $f$ ) Western blot analysis of GP96, Flotillin-1 and Tsg101 or Galectin-3 expression in the exosomes (exo) and total cell lysate (TL) from MEFs or BMDC without (-) and with $(+)$ treatment $(\gamma-$ Ir at 10 Gy or $100 \mathrm{nM}$ ActD $+100 \mu \mathrm{M}$ zVAD), $30 \mu \mathrm{g}$ of the TL and exosomal proteins derived from $0.25 \times 10^{6} \mathrm{MEFs}$ or from $3 \times 10^{6} \mathrm{BMDC}$ were loaded; anti-actin is used as control for equal loading in the TL. (c, $\mathbf{g}$ ) Western blot analysis with anti-Flotillin-1 of a continuous sucrose gradient of proteins from purified exosomes secreted by MEFs (c) or BMDCs $(\mathbf{g})$. (d, h) Western blot analysis of TSAP6, P21 and PARP cleavage on MEFs (d) or BMDCs (h) TL without $(-)$ and with $(+)$

d $\quad$ MEF TL

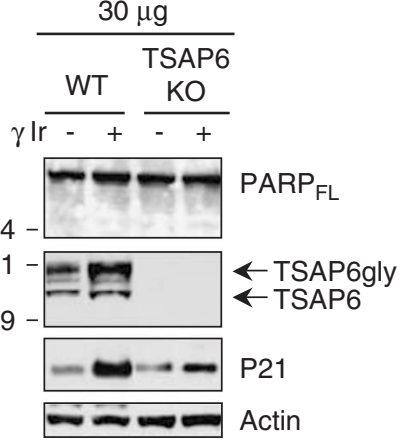

h

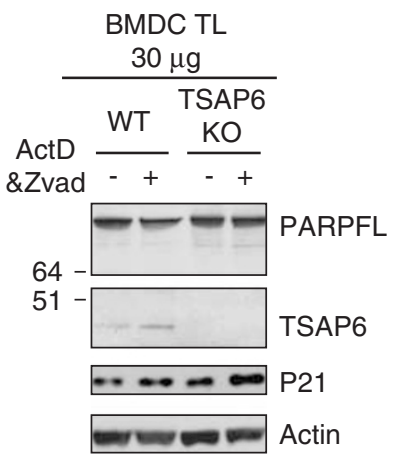


a
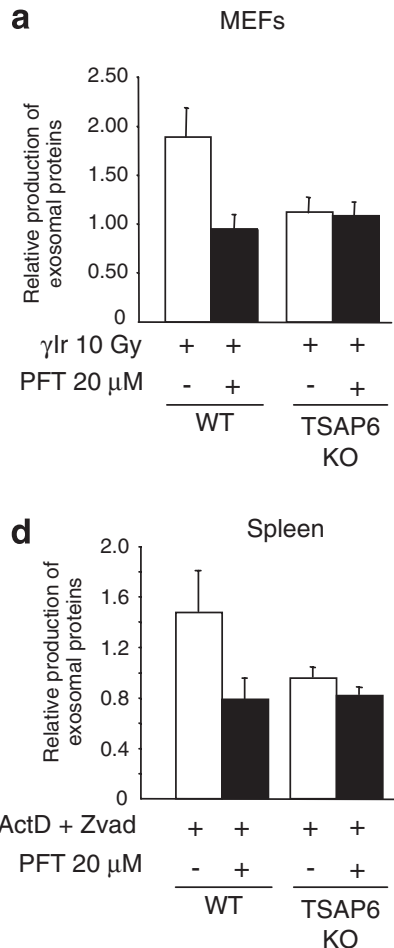

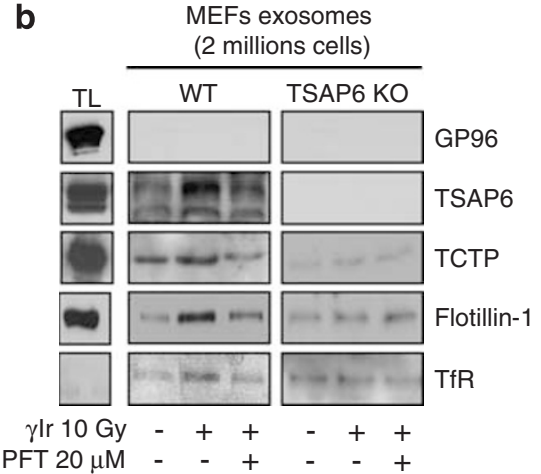

C

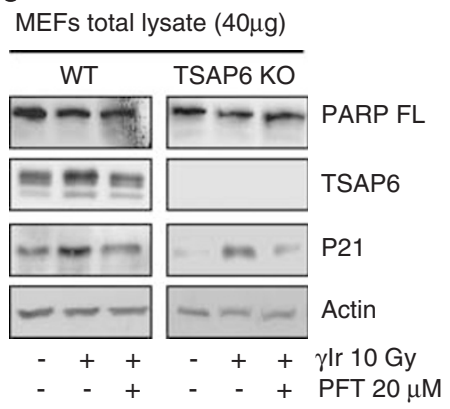

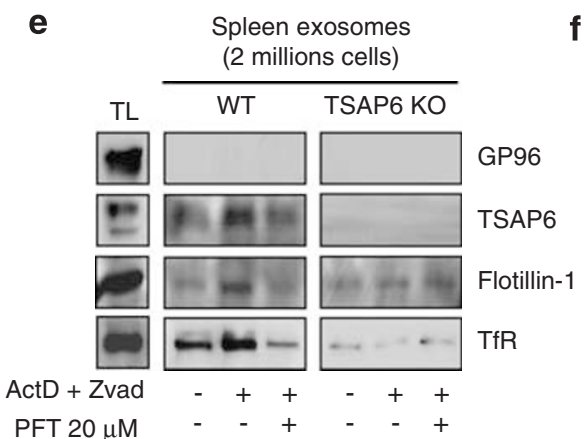

f Spleen total lysate (40ug)

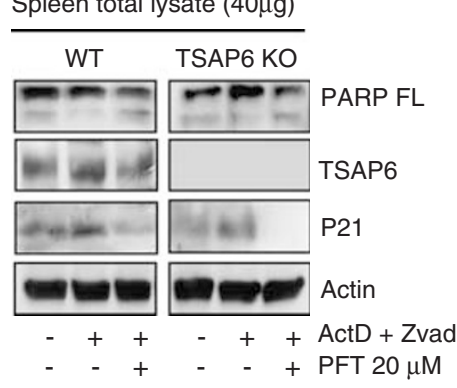

Figure 6 Exosomal protein secretion in mouse embryo fibroblasts (MEFs) and splenocytes from wild-type and tumor suppressor-activated pathway 6 (TSAP6) knockout (KO) mice after DNA damage and Pifithrin- $\alpha$ treatment. (a, d) Quantitative analysis of exosomal protein production by, respectively, MEFs (a) or spleen cells (d) from wild-type (WT) and TSAP6 KO mice. The results are presented as the ratio between exosomal protein production after $\gamma$ irradiation (MEFs) or ActD (spleen cells) treatment versus the same cells not treated. (b, e) Western blot analysis of GP96, TSAP6, TCTP (for MEFs), Flotillin-1 and Tf-R expression in the exosomes of WT and TSAP6 KO cells treated by DNA-damaging agents $(-,+)$ and Pifithrin- $\alpha(-,+)$. (c, f) Western blot analysis of PARP cleavage, TSAP6, P21 and actin in the total cell lysate of WT and TSAP6 KO cells treated by DNA-damaging agents $(-,+)$ and Pifithrin- $\alpha(-,+)$

the sorting of some proteins. Indeed, instead of increasing after irradiation, Tsg101 decreases in TSAP6 KO cells, whereas Flotillin-1 remains stable (Figure $5 \mathrm{~b}$ ). This can be due to the fact that Flotillin-1 is typically a lipid raft-associated protein and is sorted as such, together with tetraspan proteins, whereas Tsg101 is part of the ESCRT (endosomal sorting complex required for transport) machinery. The exosomes were tested for their migration on a sucrose density gradient as this is the parameter that most accurately defines the exosomal fraction (Figure $5 \mathrm{c}$ and $\mathrm{g}$ ), it is thus a qualitative parameter. All the experiments were carried out before there was any detectable sign of apoptosis that could contaminate the exosomes with cellular debris and apoptotic bodies, and no significant cleavage of the PARP was detected (Figure 5d and $\mathrm{h}$ ). Moreover, the experiments on BMDCs were carried out in the presence of $z \mathrm{VAD}$-fmk when actinomycinD was added, and this again to avoid any induction of apoptosis. As control, we used GP96; as shown in Figure 5b and f, there is no contamination of the exosomal fraction with GP96, indicating that there is no cell death or unspecific protein leakage occurring. Induction of TSAP6 was monitored by western blot analysis, and activation of p53 was assessed by analyzing induction of P21 expression (Figure $5 d$ and h). This level of induction of TSAP6 and P21 is more pronounced in the MEFs (Figure 5d) than in the BMDCs (Figure 5h) due to the difference in cellular system, DNA-damaging agent (irradiation or actinomycinD) and time of incubation post induction.

We further used Pifithrin- $\alpha$ as p53 inhibitor ${ }^{28}$ to assess whether or not TSAP6 exosomes are p53 dependent (Figure 6). In two different cellular systems, MEFs and spleen cells, following irradiation or actinomycinD treatment, Pifithrin- $\alpha$ significantly inhibited the production of exosomes in WT cells (Figure $6 \mathrm{a}$ and $\mathrm{d}$ ). The expression of specific exosomal proteins such as TSAP6, TCTP, Flotillin-1 and TF-R was activated following DNA damage and strongly inhibited by Pifithrin- $\alpha$ in WT cells only (Figure $6 b$ and e). The effect of Pifithrin- $\alpha$ was assessed by monitoring the inhibition of expression of P21 (Figure 6c and f). In the MEFs no sign of apoptosis was present (PARP; Figure 6c). In the spleen cells, there was a minor effect on PARP cleavage (PARP; Figure 6f), but most importantly, no leakage of GP96 into the exosomal fraction was detected (Figure 6e). These results indicate that p53 activation by DNA damage induces an increased secretion of exosomes and that this process is TSAP6 dependent.

\section{Discussion}

It was unexpected that p53 could control the protein secretory machinery, as previous reports described p53 target genes that were bona fide secreted proteins such as thrombospondin-1, 
IGF-BP3, plasminogen activator and maspin, but none of them regulators of the protein secretory pathways. ${ }^{29-33} \mathrm{We}$ had previously shown that TSAP6 facilitates the secretion of TCTP through the nonclassical pathway. ${ }^{6}$ In the present study, we aimed at getting a more physiological picture of the role of TSAP6 as a potential regulator of the exosomal secretion pathway, and analyzed whether it was part of an essential p53-regulated process.

We first show herein that a fraction of TSAP6 is glycosylated and colocalizes with Tf-R and EEA1, suggesting that TSAP6 regulates the endosomal pathway. TSAP6 also colocalizes with TGN compartment responsible for the biogenesis of exosomes. This distribution reflects a central role of TGN in protein secretion by both classical and nonclassical routes. Several reports indicate that the translocation site of galectins might be related to exosome biogenesis and that exosome formation is highly connected with TGN. Very interestingly, it has recently been demonstrated in epithelial cells that galectin-3, a protein also identified in exosomes secreted by dendritic cells, ${ }^{34}$ is involved in apical sorting of glycoproteins from the TGN through binding to their carbohydrate residues. ${ }^{35}$ It is thus tempting to envision TGN as a compartment where alternative secretion of proteins could occur. Although formation of exosomes is often presented as a process taking place in late endosomal structures, there is strong evidence that depending on the cell type, exosomes can be formed from a recycling endosomal/TGN compartment. ${ }^{25,36-38}$ The 'Trojan exosome hypothesis' has been proposed in the biogenesis of retroviruses. ${ }^{14}$ This hypothesis is partly based on similarities in the biogenesis of viral and exosomal particles, especially the implication of the ESCRT machinery. Targeting of the HIV type 1 envelope to the TGN through binding to TIP47 has been demonstrated to be essential for env incorporation into virions and infectivity. ${ }^{39}$ Altogether these data are consistent with ones' expectations from a protein, such as TSAP6, to fulfill a regulatory role in the fate of exosomes.

The most striking phenotype of TSAP6 $\mathrm{KO}$ mice is the microcytic anemia with anisocytosis and an augmented osmotic fragility. One explanation for the microcytosis is that TSAP6 is a ferrireductase. ${ }^{5}$ Our results suggest that there is an alternative explanation for the TSAP6-/- phenotype, namely the one provided by the above-discussed data on the localization of TSAP6 and the biological connection between the endosomal/TGN compartment and exosomes. The absence of TSAP6 causes a deregulation of these compartments, resulting in a severe deficiency in exosome formation delaying the maturation of bone marrow erythroblasts and peripheral blood reticulocytes, leading to smaller cells retaining the Tf-R instead of expelling it. A significantly higher percentage of reticulocytes and higher levels of Tf-R in TSAP6 $\mathrm{KO}$ mice prior to $\mathrm{PHZ}$ treatment is consistent with this view. Moreover, following induction of anemia by phenylhydrazine, a clear defect in reticulocyte maturation was observed in absence of TSAP6. Altogether these data indicate that the anemic phenotype is due, at least to a large extent, to an improper reticulocyte maturation with impaired jettison of their $\mathrm{Tf}-\mathrm{R}$ which is, as previously described, an exosomal process.

We finally questioned whether TSAP6 is part of an essential p53-regulated process. We therefore investigated whether
TSAP6 per se is necessary for the secretion of exosomes following p53 activation. Our results indicate that in different cellular systems (MEFs, BMDCs and spleen cells) there is a complete absence of response to p53 for exosomal protein secretion in TSAP6-/- mice. More than 20 years separate by now the discovery of exosomes and TSAP6, the first described protein regulating their production. Our results indicate that one function of TSAP6 in regulating exosome production is to expel from cells proteins that are not necessary anymore for their further cell maturation and survival. Another example of a protein that is expelled from the cells, in this case through its interaction with TSAP6, is TCTP. ${ }^{6}$ This is an antiapoptotic factor and thus, reducing its intracellular load in cancer cells renders them more prone to apoptosis. Here the exosome may function as a 'detoxifier' of the cell in a p53-regulated process. A second function of the exosomes and TSAP6 could be to communicate with the extracellular environment in signaling abnormal intracellular events. As a consequence p53, by up-regulation of TSAP6, could influence cell fate in a non-cell autonomous manner and control the 'bystander effect'. In patients with a loss of p53 function, this would mean that exosomes do not vehiculate the messages anymore and that destruction of cancer cells by the immune system would be inefficient. Altogether this opens a new area of research where we could learn how the p53TSAP6 pathway signals the status of a cell to its surrounding.

\section{Materials and Methods}

Generation of TSAP6 knockout mice. To delete the second and the third exons of murine TSAP6 gene, a targeting vector was constructed as follows: a genomic region containing the second and third coding exons of the murine TSAP6 was amplified by PCR using following primers containing a Xhol site on the forward primer and a Sacll site on the reverse primer: forward: $5^{\prime}$-CCGCTCGAG CATATATTTTGCATGCGCCC-3', reverse: $5^{\prime}$-TTCCCGCGGGCCATCCA GGCCA TGACATTG-3' (Figure 1a). The Xhol/Sacll fragment was inserted into a pBluescript (pBS) vector. A 46 bp polynucleotide containing a LoxP site floxed by two Nhel sites was added $404 \mathrm{bp}$ upstream exon 2 in the unique Spel. Finally, a neomycin resistance gene floxed by two LoxP sites was added 533 bp downstream exon 3 . This construct was linearized and introduced into C57Bl6 ES cells to obtain recombinant mice in which the exons 2 and 3 are excised upon Cre-mediated recombination. Correctly targeted ES clones (Tsap $6^{\mathrm{Neo-R/+}}$ ) were obtained, as assessed by PCR and Southern blot, and Cre-mediated excision of exons 2-3 was achieved upon electroporation of a Cre-recombinase expression vector in those cells. One clone that showed proper deletion of exons 2-3 $\left(\right.$ Tsap $\left.^{\Delta 2-3 /+}\right)$, as identified by PCR analysis, was used to establish a Tsap6-mutant mouse line. Mice were genotyped using a PCR-based approach using primers F1, R1 and R2

1. F1 5'-CTGTCCCATGTCCTCTTAAGTTATC-3';

2. R1 $5^{\prime}$-AGCTCAGAAATTAGAGGGAGAGGAT-3'and

3. R2 5'-TGAACCCCAGAATACATGAATAAGT-3' (Figure 1a).

Heterozygous $\left(T \operatorname{sap} 6^{\Delta 2-3 /+}\right)$ and homozygous $\left(T s a p 6^{\Delta 2-3 / \Delta 2-3}\right.$; thereafter referred to as Tsap6-1-) mice for this mutation are viable and fertile. For all experiments, TSAP6 WT and KO mice were obtained by crossing two TSAP6 heterozygotes. Mice of the same litter or of the same age were used in each experiment. Analysis of the phenotype was performed on 24 mice: $5 \mathrm{KO}$ males, $7 \mathrm{KO}$ females, 6 control WT males and females. WT and KO lines have a $75 \%$ C57BI6-mixed genetic background. All experiments were carried out in accordance with the European Communities Council Directive of 24 November 1986.

BMDCs were cultured in IMDM (Sigma) with 10\% endotoxin-free FCS (BioWest), $1 \%$ L-glutamine, $500 \mu \mathrm{M} \beta$-mercaptoethanol, antibiotics and $30 \%$ conditioned medium from $\mathrm{J} 558$ cells (a GM-CSF-secreting plasmacytoma kindly provided by Dr. C. Théry). Briefly, precursors of BMDCs, were obtained by flushing femurs and tibias with IMDM using a 25-gauge needle. Cells were grown during 8-13 days to induce differentiation into BMDC (expression of CD11c). 
Immunofluorescence analysis. MEFs were fixed for $20 \mathrm{~min}$ at room temperature with paraformaldehyde $4 \%$ in PBS, permeabilized for 10 min with Triton $\mathrm{X}-1000.2 \%$ in PBS, and stained with primary antibodies including: polyclonal antiTSAP6 at 1/100, sheep anti-TNG38 (Serotec) at 1/100 and mouse anti-Tf-R H68.4 (Zymed) at 1/50. The respective secondary antibodies were: anti-rabbit Alexa 594 (red), anti-goat Alexa 488 (green) or anti-mouse Alexa 488 (green) antibodies at $1 / 4000$ (Molecular Probes). Confocal imaging was performed using a Zeiss LSM $5 \mathrm{~Pa}$ confocal microscope.

FACS analysis. Freshly isolated reticulocytes from $\mathrm{PHZ}$-treated or control mice (WT and TSAP6 KO) were washed three times in PBS and then immunostained with allophycocyanin (APC)-conjugated anti-Ter119 (1:200; BD Pharmingen) and phycoerythrin (PE)-conjugated anti-CD71 (1:200; BD Pharmingen) antibodies, as previously described. The same protocol was used on erythroblasts, isolated from bone marrow of WT or TSAP6 KO mice as previously described.

For mice that were not PHZ treated (WT and TSAP6 KO), red cells were collected, washed in PBS. As for PHZ-treated mice, Tf-R quantity was determined by using PE-conjugated anti-CD71 (1:200; BD Pharmingen) antibody. ${ }^{40}$

For spleen immunophenotyping and apoptosis assay, spleens were first ground and cells washed three times in PBS. Immunophenotyping was determined by using a mix of APC-conjugated anti-Ter119, allophycocyanin Cy7 (APC-Cy7)-conjugated anti-B220, PE-conjugated anti-CD11b (1:200; BD Pharmingen) antibodies or a mix of allophycocyanin (APC)-conjugated anti-Ter119, PE-conjugated anti-CD4, phycoerythrin Cy7 (PE-Cy7)-conjugated anti-CD8 (1:200; BD Pharmingen) antibodies. In both the cases, apoptosis was determined by adding FITCconjugated Annexin V (Sigma Lab.).

In each case, fluorescence was finally monitored by flow cytometry on a FACSCanto instrument (BD Pharmingen) operating with FlowJo 7.2 software (Tree Star Inc.)

Isolation of exosomes. For exosome production, cells were cultured in complete medium. This medium was depleted of contaminating vesicles and protein aggregates by overnight centrifugation at $100000 \times g$ in OPTIMA ${ }^{\mathrm{TM}}$ MAX-E ultracentrifuge (Beckman Coulter). Recovery of $2.10^{6}$ MEFs culture medium for exosomal analysis was carried out $24 \mathrm{~h}$ after a $10 \mathrm{~Gy} \gamma$-irradiation (IBL637 irradiator $\mathrm{Cs}^{137}$ ). BMDCs were plated at $1.5 \times 10^{6}$ cells per ml with $100 \mathrm{nM}$ actinomycin D (Sigma) and $100 \mu \mathrm{M}$ zVAD-fmk (benzyloxycarbonyl-Val-Ala-Asp-fluoromethylketon; Sigma). Recovery of the BMDCs medium was done $6 \mathrm{~h}$ after treatment. The culture media were placed on ice, centrifuged at $450 \times g$ to sediment the cells and filtered on $0.22 \mu \mathrm{M}$ to remove cellular debris. Exosomes were separated from the supernatant by centrifugation at $100000 \times g$ for $70 \mathrm{~min}$ at $4^{\circ} \mathrm{C}$. The exosome pellets were washed with a large volume of PBS, centrifuged at $100000 \times g$ for $70 \mathrm{~min}$ and resuspended in $50 \mu \mathrm{l}$ of PBS. Exosomes $(30 \mu \mathrm{g})$ were further purified by a $16 \mathrm{~h}$ ultracentrifugation at $100000 \times g$ on a linear $0.25-2.5 \mathrm{M}$ sucrose gradient. Flotation of exosomes on a continuous gradient was performed in a TLA110 rotor. Fractions of the gradient $(500 \mu \mathrm{l})$ were diluted in $3 \mathrm{ml}$ of PBS and ultracentrifuged for $2 \mathrm{~h}$ at $200000 \times \mathrm{g}$. Pellets of each fraction were analyzed by western blot. The preparation of exosomes derived from the spleen cells were performed as described above for the other cellular systems. Pifithrin- $\alpha$ (Sigma) was used as p53 inhibitor at a concentration of $20 \mu \mathrm{M}$ prior to induction of the DNA damage.

Pathology, hematology and blood biochemistry. Mice (4-month old), 6 TSAP6 KO and 6 WT of each sex, were subjected to systematic necropsy. Body weight and length, liver, kidney, spleen, heart and paragenital fat weight were recorded. Organs were fixed $24 \mathrm{~h}$ in $4 \%$ buffered formaldehyde and embedded in paraffin. Hematoxylin and eosin-stained sections were prepared from tissues of three TSAP6 KO and one WT of each sex. Peripheral blood counts and blood biochemistry were analyzed using standard procedures. Data were analyzed using unpaired Student's $t$-test or repeated measures analysis of variance with one between factor (genotype) and one within factor (time). Qualitative parameters (e.g. clinical observations) were analyzed using $\chi^{2}$-tests. The level of significance was set at $P<0.05$. For the osmotic fragility $4 \mu$ l of peripheral blood were incubated $10 \mathrm{~min}$ at room temperature in $1 \mathrm{ml}$ of hyperosmotic solution (sucrose/PBS from 400 to 1200 mosM). Hemoglobin concentration in supernatant was measured at OD $535 \mathrm{~nm}$ after centrifugation. Primary MEFs were obtained from 12.5 days old embryos using established procedures. TUNEL analysis was performed as described before. ${ }^{2}$
Reticulocytes. Anemia was induced in mice in order to activate the production reticulocytes. For this purpose, mice were treated with $100 \mu \mathrm{l} \mathrm{PHZ}$ at $6 \mathrm{mg} / \mathrm{ml}$ in $150 \mathrm{mM} \mathrm{NaCl}$ buffer for 2 consecutive days. After 4 days of recovery, total blood was collected from anemic mice in heparinized tubes by cardiac punction. Reticulocytes were separated from erythrocytes on a Percoll gradient $(1.1-1.123 \mathrm{~g} / \mathrm{ml})$. The reticulocytes were recovered at the top of the gradient, washed and seeded in RPM medium containing $5 \mathrm{mM}$ L-glutamine, $5 \mathrm{mM}$ adenosine and $10 \mathrm{mM}$ inosine. After 3 days culture at $37^{\circ} \mathrm{C}$, the reticulocytes had differentiated into erythrocytes as assessed by cresyl blue coloration.

Bone marrow analysis. A bone marrow analysis was performed on WT and TSAP6 KO mice using standard procedures, by flushing the femoral bones and a May-Grünwald-Giemsa staining.

Antibodies. Antibodies against the N-terminal peptide (MSGEMDKPLI SRRLVDSDGS) of mouse TSAP6 were generated in rabbits, affinity purified and used at 1/2000 for western blotting. Other primary antibodies used: anti-PARP H250 (Santa Cruz) at 1/1000; anti-Tf-R H68.4 (Zymed) at 1/1000; anti-Flotillin-1 (BD Biosciences) at 1/1000; anti-p21 F5 (Santa Cruz) at 1/500; anti-Actin I19 and antiPCNA FL-261 (Santa Cruz) at 1/1000, GP96 (1/500) from Stressgen, Tsg101 M-19 (1/500) and Galectin-3 D-20 (1/500) from Santa Cruz. Secondary antibodies: antimouse HRP linked (Santa-Cruz), anti-goat HRP linked (Calbiochem) and anti-rabbit HRP linked (Calbiochem) were used at 1/5000.

Glycosylation and dimerization analysis. Glycosylation analysis was performed using Glycoprotein deglycosylation kit (Calbiochem) following manufacturer's recommendations. For the dimerization analysis NIH3T3 cells were co-transfected with the mouse constructs TSAP6-Flag and TSAP6-GFP. $24 \mathrm{~h}$ after transfection cells were lysed in IP buffer ( $50 \mathrm{mM}$ Tris pH 7.5, $150 \mathrm{mM} \mathrm{NaCl}, 1 \%$ NP40). $200 \mu \mathrm{g}$ of proteins were incubated overnight at $4^{\circ} \mathrm{C}$ with anti-Flag antibody coated agarose beads (mouse clone M2 Sigma). Mouse serum plus agarose beads were used as negative control. After 3 washes in IP buffer, beads were heated at $95^{\circ} \mathrm{C}$ for $5 \mathrm{~min}$ in sample buffer and loaded on a SDS polyacrylamide gel and western blots were performed and hybridized with anti-GFP antibodies (Santa Cruz).

siRNA. Murine TSAP6 RNA duplex were synthesized by Dharmacon: siRNA1: 3'-GACGTTCTACAGCCATACA-5', SIRNA2: $3^{\prime}$-AGCCATACATTCGGAAAGA-5' and siRNA3: $3^{\prime}$-TCAGCACCGCCAGTCTAAT-5'. Human TCTP siRNA duplex was used as control.

Acknowledgements. This study is part of the $\mathrm{PhD}$ thesis of Alexandra Lespagnol (Université Paris VII), and Dominique Duflaut (Université Paris XI). We are grateful to Tania Sorg and Daniel Metzger for their advice in the generation of the TSAP6 knockout mice, to Alain Schmitt, Marie-Claude Gendron, Myriam Garfa, and Evelyne Souil for their expertize, Naomi Taylor for her scientific and technical help and Marie-Claude Guillemin for the bone marrow analysis and Pierre Hainaut for his constructive comments. We thank Séverine Beaucourt for her involvement in the initial experiments (see PhD thesis online: These_Severine_Beaucourt.pdf) $\mathrm{CB}$ is supported by grants from the Belgian Foundation for Cancer Research. This work was supported by grants from ARC (no. 3444) to MV, Conticanet (Network of Excellence) and Eureka from the European Commission to AT and RA.

Competing interests statement

The authors declare that they have no competing financial interests.

1. Amson RB, Nemani M, Roperch JP, Israeli D, Bougueleret L, Le Gall I et al. Isolation of 10 differentially expressed cDNAs in p53-induced apoptosis: activation of the vertebrate homologue of the Drosophila seven in absentia gene. Proc Natl Acad Sci USA 1996; 93 . 3953-3957.

2. Roperch JP, Alvaro V, Prieur S, Tuynder M, Nemani M, Lethrosne F et al. Inhibition of presenilin 1 expression is promoted by p53 and p21WAF-1 and results in apoptosis and tumor suppression. Nat Med 1998; 4: 835-838.

3. Passer BJ, Nancy-Portebois V, Amzallag N, Prieur S, Cans C, Roborel de Climens A et al. The p53-inducible TSAP6 gene product regulates apoptosis and the cell cycle and interacts with Nix and the Myt1 kinase. Proc Natl Acad Sci USA 2003; 100: 2284-2289.

4. Sanchez-Pulido L, Rojas AM, Valencia A, Martinez AC, Andrade MA. ACRATA: a novel electron transfer domain associated to apoptosis and cancer. BMC Cancer 2004; 4: 98. 
5. Ohgami RS, Campagna DR, Greer EL, Antiochos B, McDonald A, Chen $J$ et al. Identification of a ferrireductase required for efficient transferrin-dependent iron uptake in erythroid cells. Nat Genet 2005; 37: 1264-1269.

6. Amzallag N, Passer BJ, Allanic D, Segura E, Thery C, Goud B et al. TSAP6 facilitates the secretion of translationally controlled tumor protein/histamine-releasing factor via a nonclassical pathway. J Biol Chem 2004; 279: 46104-46112.

7. Tuynder M, Susini L, Prieur S, Besse S, Fiucci G, Amson R et al. Biological models and genes of tumor reversion: cellular reprogramming through tpt1/TCTP and SIAH-1. Proc Natl Acad Sci USA 2002; 99: 14976-14981.

8. Susini L, Besse S, Duflaut D, Lespagnol A, Beekman C, Fiucci G et al. TCTP protects from apoptotic cell death by antagonizing bax function. Cell Death Differ 2008 Advance online publication 15 February.

9. Rubartelli A, Cozzolino F, Talio M, Sitia R. A novel secretory pathway for interleukin-1 beta a protein lacking a signal sequence. EMBO J 1990; 9: 1503-1510.

10. Hughes RC. Secretion of the galectin family of mammalian carbohydrate-binding proteins. Biochim Biophys Acta 1999; 1473: 172-185.

11. Nickel W. The mystery of nonclassical protein secretion. A current view on cargo proteins and potential export routes. Eur J Biochem 2003; 270: 2109-2119.

12. Pan BT, Johnstone RM. Fate of the transferrin receptor during maturation of sheep reticulocytes in vitro: selective externalization of the receptor. Cell 1983; 33: 967-978.

13. Johnstone RM. Revisiting the road to the discovery of exosomes. Blood Cells Mol Dis 2005 34: $214-219$

14. Gould SJ, Booth AM, Hildreth JE. The Trojan exosome hypothesis. Proc Natl Acad Sci USA 2003; 100: 10592-10597.

15. Nguyen DG, Booth A, Gould SJ, Hildreth JE. Evidence that HIV budding in primary macrophages occurs through the exosome release pathway. J Biol Chem 2003; 278: 52347-52354.

16. Raposo G, Nijman HW, Stoorvogel W, Liejendekker R, Harding CV, Melief CJ et al. B lymphocytes secrete antigen-presenting vesicles. J Exp Med 1996; 183: 1161-1172.

17. Thery C, Zitvogel L, Amigorena S. Exosomes: composition, biogenesis and function. Nat Rev Immunol 2002; 2: 569-579.

18. Zitvogel L, Regnault A, Lozier A, Wolfers J, Flament C, Tenza D et al. Eradication of established murine tumors using a novel cell-free vaccine: dendritic cell-derived exosomes. Nat Med 1998; 4: 594-600.

19. Yu X, Harris SL, Levine AJ. The regulation of exosome secretion: a novel function of the p53 protein. Cancer Res 2006; 66: 4795-4801.

20. de Gassart A, Geminard C, Hoekstra D, Vidal M. Exosome secretion: the art of reutilizing nonrecycled proteins? Traffic 2004; 5: 896-903.

21. Ross SR, Schofield JJ, Farr CJ, Bucan M. Mouse transferrin receptor 1 is the cell entry receptor for mouse mammary tumor virus. Proc Natl Acad Sci USA 2002; 99: 12386-12390.

22. Burzyn D, Rassa JC, Kim D, Nepomnaschy I, Ross SR, Piazzon I. Toll-like receptor 4-dependent activation of dendritic cells by a retrovirus. J Virol 2004; 78: 576-584.

23. Aisen P. Transferrin receptor 1. Int J Biochem Cell Biol 2004; 36: 2137-2143.
24. Vidal $M$, Mangeat $P$, Hoekstra $D$. Aggregation reroutes molecules from a recycling to a vesicle-mediated secretion pathway during reticulocyte maturation. J Cell Sci 1997; 110 (Part 16): 1867-1877.

25. Savina A, Vidal M, Colombo Ml. The exosome pathway in K562 cells is regulated by Rab11. J Cell Sci 2002; 115: 2505-2515.

26. Clayton A, Turkes A, Navabi H, Mason MD, Tabi Z. Induction of heat shock proteins in B-cell exosomes. J Cell Sci 2005; 118: 3631-3638.

27. Lancaster GI, Febbraio MA. Exosome-dependent trafficking of HSP70: a novel secretory pathway for cellular stress proteins. J Biol Chem 2005; 280: 23349-23355.

28. Komarov PG, Komarova EA, Kondratov RV, Christov-Tselkov K, Coon JS, Chernov MV et al. A chemical inhibitor of p53 that protects mice from the side effects of cancer therapy. Science 1999; 285: 1733-1737.

29. Dameron KM, Volpert OV, Tainsky MA, Bouck N. Control of angiogenesis in fibroblasts by p53 regulation of thrombospondin-1. Science 1994; 265: 1582-1584.

30. Kunz C, Pebler S, Otte J, von der Ahe D. Differential regulation of plasminogen activator and inhibitor gene transcription by the tumor suppressor p53. Nucleic Acids Res 1995; 23: 3710-3717.

31. Buckbinder L, Talbott R, Velasco-Miguel S, Takenaka I, Faha B, Seizinger BR et al. Induction of the growth inhibitor IGF-binding protein 3 by p53. Nature 1995; 377: 646-649.

32. Komarova EA, Diatchenko L, Rokhlin OW, Hill JE, Wang ZJ, Krivokrysenko VI et al. Stressinduced secretion of growth inhibitors: a novel tumor suppressor function of p53. Oncogene 1998; 17: 1089-1096.

33. Zou Z, Gao C, Nagaich AK, Connell T, Saito S, Moul JW et al. p53 regulates the expression of the tumor suppressor gene maspin. J Biol Chem 2000; 275: 6051-6054.

34. Rodriguez-Boulan E, Kreitzer G, Musch A. Organization of vesicular trafficking in epithelia. Nat Rev Mol Cell Biol 2005; 6: 233-247.

35. Delacour D, Cramm-Behrens $\mathrm{Cl}$, Drobecq $\mathrm{H}$, Le Bivic A, Naim HY, Jacob R. Requirement for galectin-3 in apical protein sorting. Curr Biol 2006; 16: 408-414.

36. Thery C, Boussac M, Veron P, Ricciardi-Castagnoli P, Raposo G, Garin J et al. Proteomic analysis of dendritic cell-derived exosomes: a secreted subcellular compartment distinct from apoptotic vesicles. J Immunol 2001; 166: 7309-7318

37. Bonifacino JS. The GGA proteins: adaptors on the move. Nat Rev Mol Cell Biol 2004; 5 : 23-32.

38. Mallet WG, Maxfield FR. Chimeric forms of furin and TGN38 are transported with the plasma membrane in the trans-Golgi network via distinct endosomal pathways. J Cell Biol 1999; 146: 345-359.

39. Blot G, Janvier K, Le Panse S, Benarous R, Berlioz-Torrent C. Targeting of the human immunodeficiency virus type 1 envelope to the trans-Golgi network through binding to TIP47 is required for env incorporation into virions and infectivity. J Virol 2003; 77 : 6931-6945.

40. Blanc L, Barres C, Bette-Bobillo P, Vidal M. Reticulocyte-secreted exosomes bind natural IgM antibodies: involvement of a ROS-activatable endosomal phospholipase iPLA2. Blood 2007; 110: 3407-3416.

Supplementary Information accompanies the paper on Cell Death and Differentiation website (http://www.nature.com/cdd) 